CUBO A Mathematical Journal

Vol.22, $N^{\mathrm{O}}$ 01, (39-53). April 2020

\title{
A sufficiently complicated noded Schottky group of rank three
}

\author{
RubÉN A. Hidalgd 1 \\ Departamento de Matemática y Estadística, \\ Universidad de La Frontera, Temuco, Chile. \\ ruben.hidalgo@ufrontera.cl
}

\begin{abstract}
In 1974, Marden proved the existence of non-classical Schottky groups by a theoretical and non-constructive argument. Explicit examples are only known in rank two; the first one by Yamamoto in 1991 and later by Williams in 2009. In 2006, Maskit and the author provided a theoretical method to construct non-classical Schottky groups in any rank. The method assumes the knowledge of certain algebraic limits of Schottky groups, called sufficiently complicated noded Schottky groups. The aim of this paper is to provide explicitly a sufficiently complicated noded Schottky group of rank three and explain how to use it to construct explicit non-classical Schottky groups.
\end{abstract}

\section{RESUMEN}

En 1974, Marden demostró la existencia de grupos de Schottky no-clásicos con un argumento teórico y no-constructivo. Se conocen ejemplos explícitos solo en rango dos; el primero por Yamamoto en 1991 y después por Williams en 2009. En 2006, Maskit y el autor entregaron un método teórico para construir grupos de Schottky no-clásicos en cualquier rango. El método asume el conocimiento de ciertos límites algebraicos de grupos de Schottky, llamados grupos de Schottky anodados suficientemente complicados. El objetivo de este paper es dar un grupo de Schottky anodado suficientemente complicado explícitamente de rango tres y explicar cómo usarlo para construir grupos de Schottky no-clásicos explícitos.

Keywords and Phrases: Riemann surfaces; Schottky groups.

2010 AMS Mathematics Subject Classification: 30F40, 30F10.

${ }^{1}$ Partially supported by Projects Fondecyt 1190001. 


\section{Introduction}

A Kleinian group $\mathrm{G}$ is called a Schottky group of rank $\mathrm{g} \geq 2$ if it is generated by loxodromic transformations $A_{1}, \ldots, A_{g} \in \mathrm{PSL}_{2}(\mathbb{C})$ such that there is a collection of $2 \mathrm{~g}$ pairwise disjoint simple

loops $\alpha_{1}, \alpha_{1}^{\prime}, \alpha_{2}, \alpha_{2}^{\prime}, \ldots, \alpha_{g}, \alpha_{g}^{\prime}$ on the Riemann sphere $\widehat{\mathbb{C}}$, all of them bounding a common domain $\mathcal{D}$ of connectivity $2 g$, with $A_{j}\left(\alpha_{j}\right)=\alpha_{j}^{\prime}$ and $A_{j}(\mathcal{D}) \cap \mathcal{D}=\emptyset$. The above set of generators is called geometrical and the above loops a fundamental set of loops for $\mathrm{G}$. It is well known that $\mathrm{G}$ is a free group of rank $\mathrm{g}$ and that $\mathcal{D}$ is a fundamental domain for it. In [3] Chukrow proved that every set of $\mathrm{g}$ generators of $\mathrm{G}$ is geometrical. We say that $\mathrm{G}$ is a classical Schottky group if it has a set of geometrical generators, called a classical set of generators, for which we may find a fundamental set of loops being circles. Classical Schottky groups were firstly considered by Schottky around 1882. In general, a classical Schottky group may have non-classical set of generators.

Examples of classical Schottky groups are given by the finitely generated purely hyperbolic Fuchsian groups representing a closed surface with holes [2]. Moreover, if such a Fuchsian group is a two generator group representing a torus with one hole, then every pair of generators is a classical set of generators [21].

In 1974, Marden [14] provided the existence of non-classical Schottky groups (his proof is non-constructive). In 1975, Zarrow [27] claimed to have constructed an explicit example of a nonclassical Schottky group of rank two, but it was lately noted by Sato in [22] to be incorrect. The first explicit (correct) construction was provided by Yamamoto [26] in 1991 and in 2009 another example was provided by Williams in his Ph.D. Thesis [24], both for rank two. It seems that, for rank at least three, there is not explicit example in the literature.

In [7], Maskit and the author described a theoretical method to construct non-classical Schottky groups in any rank $\mathrm{g} \geq 2$. The idea is to consider certain Kleinian groups, obtained as geometrically finite algebraic limits of Schottky groups of rank g, called sufficiently complicated noded Schottky groups of rank g (see Section 2). In this paper (see Section 3) we provide an explicit construction of a sufficiently complicated noded Schottky group of rank three and we used it to describe how to obtain a infinite family (one-dimensional) non-classical Schottky group of rank three.

To finish this introduction, and as a matter of completeness, let us mention another conjecture related to classical Schottky groups. If $\Omega$ is the region of discontinuity of a Schottky group $G$ of rank $\mathrm{g}$, then it is a connected set and $\Omega / \mathrm{G}$ is a closed Riemann surface of genus $\mathrm{g}$. Conversely, if $\mathrm{S}$ is a closed Riemann surface, then there is a Schottky group $G$ such that $S$ and $\Omega / G$ are isomorphic (Koebe's uniformization theorem). As we have the existence of non-classical Schottky group, it might be that $\mathrm{G}$ is non-classical. A conjecture (due to Bers) asserts that we may chose $\mathrm{G}$ to be classical. Some positive answers were obtained by Bobenko [1], Koebe [11, Maskit [17, Seppälä [23] (for the case in which the surface admits antiholomorphic involutions with fixed points) and 
McMullen [13] (for the case in which the surface has sufficiently many shorts geodesics). Recently, Hou [8, 9, have announced a proof of this conjecture (by using Haussdorf dimension of the limit set of Kleinian groups) and another approach in [6] (by using Belyi curves).

\section{Sufficiently complicated Noded Schottky groups}

\subsection{Noded Schottky groups}

A noded Schottky group of rank $\mathrm{g} \geq 2$ is geometrically defined as follows. Consider a collection of pairwise disjoint open topological discs $D_{1}, D_{1}^{\prime}, \ldots, D_{g}$ and $D_{g}^{\prime}$ on the Riemann sphere $\widehat{\mathbb{C}}$ so that the corresponding boundaries $\widehat{\alpha}_{1}=\partial \mathrm{D}_{1}, \widehat{\alpha}_{1}^{\prime}=\partial \mathrm{D}_{1}^{\prime}, \ldots, \widehat{\alpha}_{g}=\partial \mathrm{D}_{g}, \widehat{\alpha}_{g}^{\prime}=\partial \mathrm{D}_{g}^{\prime}$ are simple loops and they only intersect in at most finitely many points. Let $\widehat{A}_{1}, \ldots, \widehat{A}_{g}$ be Möbius transformations such that $\widehat{A}_{j}\left(\widehat{\alpha}_{j}\right)=\widehat{\alpha}_{j}^{\prime}$ and $\widehat{A}_{j}\left(D_{j}\right) \cap D_{j}^{\prime}=\emptyset$, for each $j=1, \ldots, g$. Observe that the transformation $\widehat{A}_{j}$ may only be loxodromic or parabolic. The group $\widehat{G}$, generated by these transformations, is a Kleinian group isomorphic to a free group of rank $g$. If $p$ is a point of intersection of two of the above loops, then either it is a fixed point of a parabolic transformation of $\widehat{G}$ or it has trivial $\widehat{\mathrm{G}}$-stabilizer. In the second situation, one may deform in a suitable manner these loops in order to avoid the intersection at $p$ and not adding extra intersections. In this way, two possibilities appear (up to performing the above deformation), either: (i) $\mathrm{G}$ is a Schottky group of rank $\mathrm{g}$ or (ii) there are intersection points, each of them being a fixed point of a parabolic transformation of $\widehat{\mathrm{G}}$. In case (ii) we say that $\widehat{\mathrm{G}}$ is a noded Schottky group of rank $\mathrm{g}$; we call the above set of loops $a$ fundamental set of loops and the generators a set of geometrical generators.

Remark 1. In [18, as an application of the Klein-Maskit's combination theorems, it was noted that a noded Schottky group $\widehat{G}$ is geometrically finite, that each of its parabolic elements is a conjugate of a power of one of the transformations fixing a common point of two of the fundamental loops, and that the complement $\widehat{\mathcal{D}}$ of the union of the closures of $D_{1}, D_{1}^{\prime}, \ldots, D_{g}, D_{g}^{\prime}$ is a fundamental domain for $\widehat{G}$. Different as for the case of Schottky groups, not every set of free generators of a noded Schottky group is necessarily geometrical.

\subsection{The extended region of discontinuity}

If $\Omega$ is the region of discontinuity of a noded Schottky group $\widehat{\mathrm{G}}$ of rank $\mathrm{g}$, then by adding to it the parabolic fixed points of $\widehat{\mathrm{G}}$, and with the appropriate cusped topology (see [5, 12]), we obtain its extended region of discontinuity $\Omega^{+}$; it happens that $\mathrm{S}^{+}=\Omega^{+} / \widehat{\mathrm{G}}$ is a stable Riemann surface of genus $g$. In the case that the number of nodes of $S^{+}$is $3 g-3$, we say that $\widehat{G}$ is a maximal noded Schottky group (in this case, there are exactly $2 g-2$ connected components of the complement of the nodes of $\mathrm{S}^{+}$, each one being a triple-punctured sphere). In [4] it was observed that every stable Riemann surface of genus $g$ is obtained as above; so every point of the Deligne-Mumford 

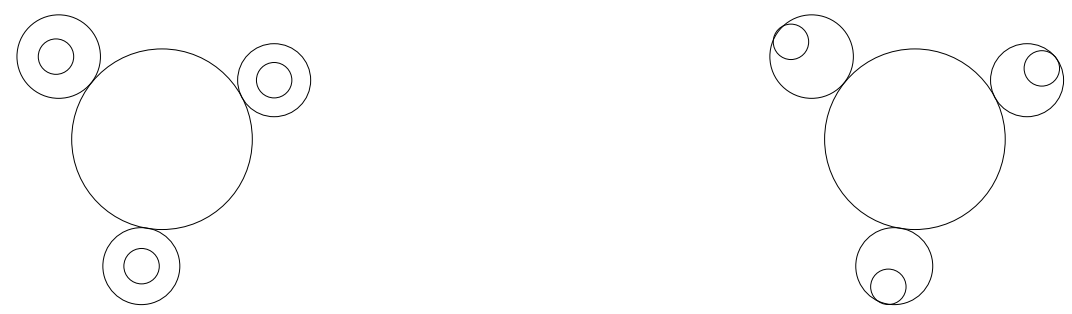

Figure 1-A stable Riemann surface of genus 3 Figure 2-A stable Riemann surface of genus 3 with 3 nodes with 6 nodes

compactification of the moduli space of genus $\mathrm{g}$ can be realized by a suitable noded Schottky group of rank $\mathrm{g}$.

\section{$2.3 \quad$ Neoclassical noded Schottky groups}

A noded Schottky group for which there is a set of geometrical generators admitting a fundamental set of loops all of which are Euclidean circles is called neoclassical; the corresponding set of generators is called a neoclassical set of generators.

In [7] it was proved that if $G$ is a noded Schottky group such that $\Omega^{+} / G$ is a stable Riemann surface as in Figure 1, then it cannot be neoclassical (this should be still true for every noded Schottky group of rank $\mathrm{g} \geq 4$ whose corresponding stable Riemann surface has $\mathrm{g}+1$ components, one being of genus zero and the others being of genus one).

\subsection{Sufficiently complicated noded Schottky groups}

The space of deformations of a Schottky group of rank $\mathrm{g}$, denoted by $\mathcal{S}_{\mathrm{alg}}$, is a subset of the representation space of the free group of rank $g$ in $\mathrm{PSL}_{2}(\mathbb{C})$, modulo conjugation. Regard $\mathbb{H}^{3}$ as being the set $\{(z, t): z \in \mathbb{C}, t>0 \in \mathbb{R}\}$. We likewise identify $\mathbb{C}$ with the boundary of $\mathbb{H}^{3}$, except for the point at infinity; that is, we identify $\mathbb{C}$ with $\{(z, t): t=0\}$.

\subsubsection{The relative conical neighbourhood of a noded Schottky group}

Let $\widehat{G}$ be a noded Schottky group of rank $g \geq 2$, with a set of geometrical generators $\widehat{A}_{1}, \ldots, \widehat{A}_{g}$, and corresponding fundamental set of loops $\widehat{\alpha}_{1}, \ldots, \widehat{\alpha}_{g}^{\prime}$, these being the corresponding boundary loops of a collection of pairwise disjoint open discs $D_{1}, \ldots, D_{g}^{\prime}$. The complement of the closures of these discs is a fundamental domain $\widehat{D}$ for $\widehat{G}$. Let $\widehat{P}_{1}, \ldots, \widehat{P}_{q}$ be a maximal set of primitive parabolic elements of $\widehat{G}$ generating non-conjugate cyclic subgroups, where $q \geq 1$ (we may assume the fix point of these parabolic transformations to be contained in the intersection of two fundamental loops). We denote by $\Omega(\widehat{\mathrm{G}})$ its region of discontinuity and by $\Omega^{+}(\widehat{\mathrm{G}})$ its extended region of discontinuity. 
Next, we proceed to recall a construction done in [7] of a one-real family of Schottky groups $\mathrm{G}^{\tau}$ whose geometric limit is $\widehat{\mathrm{G}}$.

\section{(I) The infinite shoebox construction}

For each $i=1, \ldots, q$, choose a particular Möbius transformation $H_{i}$ conjugating $\widehat{P}_{i}$ to the transformation $\mathrm{P}(z)=z+1$ and consider the renormalized group $\mathrm{H}_{i} \widehat{\mathrm{G}} \mathrm{H}_{i}^{-1}$. For this group, there is a number $\tau_{0}>1$ so that the set $\left\{|\operatorname{Im}(z)| \geq \tau_{0}\right\}$ is precisely invariant under the stabilizer $\operatorname{Stab}(\infty)$ of $\infty$ in the group $\mathrm{H}_{i} \widehat{\mathrm{G}} \mathrm{H}_{i}^{-1}$. In this normalization, for each parameter $\tau$, with $\tau>\tau_{0}$, we define the infinite shoebox to be the set $\mathrm{B}_{0, \tau}=\{(z, t):|\operatorname{Im}(z)| \leq \tau, t \leq \tau\}$. Since $\tau_{0}>1$, we easily observe that for every $\tau>\tau_{0}$, the complement of $B_{0, \tau}$ in $\mathbb{H}^{3} \cup \mathbb{C}$ is precisely invariant under $\operatorname{Stab}(\infty) \subset \mathrm{H}_{i} \widehat{\mathrm{G}} \mathrm{H}_{i}^{-1}$, where we are now regarding Möbius transformations as hyperbolic isometries, which act on the closure of $\mathbb{H}^{3}$. Then for $\widehat{G}$, the infinite shoebox with parameter $\tau$ at $z_{i}$, the fixed point of $\widehat{P}_{i}$, is $B_{i, \tau}=H_{i}^{-1}\left(B_{0, \tau}\right)$. If $\widehat{P}$ is any parabolic element of $\widehat{G}$, conjugate to some power of $\widehat{P}_{i}$, then the corresponding infinite shoebox at the fixed point of $\widehat{P}$, is given by $T\left(B_{i, \tau}\right)$, where $\widehat{P}=\mathrm{TP}_{\mathrm{i}} \mathrm{T}^{-1}$. It was observed in [19] that, for each fixed $\tau>\tau_{0}, \widehat{\mathrm{G}}$ acts as a group of conformal homeomorphisms on the expanded regular set $\mathrm{B}^{\tau}=\bigcap \widehat{\mathrm{A}}\left(\mathrm{B}_{i, \tau}\right)$, where the intersection is taken over all $\widehat{A} \in \widehat{G}$ and all $i=1, \ldots, q$. Further, $\widehat{G}$ acts as a (topological) Schottky group (in the sense of our geometrical definition) on the boundary of $B^{\tau}$. Each parabolic $\widehat{P} \in \widehat{G}$ appears to have two fixed points on the boundary of $\mathrm{B}^{\tau}$; that is, $\widehat{\mathrm{P}}$, as it acts on the boundary of $\mathrm{B}^{\tau}$, appears to be loxodromic. The flat part of $\mathrm{B}^{\tau}$ is the intersection of $\mathrm{B}^{\tau}$ with the extended complex plane $\widehat{\mathbb{C}}$. The complement of the flat part (on the boundary of $\mathrm{B}^{\tau}$ ) is the disjoint union of 3-sided boxes, where each box has two vertical sides (translates of the sets $\{\operatorname{Im}(z)= \pm \tau, 0<t<\tau\}$ ) and one horoball side (a translate of the set $\{|\operatorname{Im}(z)| \leq \tau, t=\tau\}$ ). For each $i=1 \ldots, q$ and for each integer $n \geq 1$, we set $B_{i, \tau, n}=H_{i}^{-1}\left(B_{0, \tau} \cap\{|\operatorname{Re}(z)| \leq n\}\right)$ and $B^{\tau, n}=\bigcap_{\widehat{A} \in \widehat{G}} \widehat{A}\left(B_{i, \tau, n}\right)$. The truncated flat part of $B^{\tau, n}$ is the intersection $B^{\tau, n} \cap \widehat{\mathbb{C}}$. The boundary of the truncated flat part near a parabolic fixed point, renormalized so as to lie at $\infty$, is a Euclidean rectangle. Let us renormalize $\widehat{\mathrm{G}}$ so that $\infty \in \Omega(\widehat{G})$. Then, for each $\tau>\tau_{0}$, there is a conformal map $f^{\tau}$, mapping the boundary of $B^{\tau}$ to $\widehat{\mathbb{C}}$, and conjugating $\widehat{\mathrm{G}}$ onto a Schottky group $\mathrm{G}^{\tau}$, where $\mathrm{f}^{\tau}$ is defined by the requirement that, near $\infty, f^{\tau}(z)=z+\mathrm{O}\left(|z|^{-1}\right)$. The group $\mathrm{G}^{\tau}$ depends on the choice of the Möbius transformations $H_{1}, \ldots, H_{q}$ as well as on the choice of $\tau$. The elements $A_{1}^{\tau}=f^{\tau} \widehat{A}_{1}\left(f^{\tau}\right)^{-1}, \ldots, A_{g}^{\tau}=f^{\tau} \widehat{A}_{g}\left(f^{\tau}\right)^{-1}$ provide a set of free generators for the Schottky group $\mathrm{G}^{\tau}$.

\section{(II) Vertical projection loops}

Next, we proceed to construct a fundamental set of loops for $\mathrm{G}^{\tau}$ for the above generators in terms of the fundamental set of loops for $\widehat{G}$. In [19] it was shown that, with the above normalization, $f^{\tau}$ converges to the identity I, uniformly on compact subsets of $\Omega(\widehat{G})$, and, for each $j \in\{1, \ldots, g\}$, $A_{j}^{\tau}$ converges to $\widehat{A}_{j}$, as $\tau \rightarrow \infty$. In particular, if we fix $\tau_{0}$, and fix $n$, then $f^{\tau} \rightarrow$ I uniformly on 
compact subsets of the truncated flat part of $\mathrm{B}^{\tau_{0}, n}$. The boundary of $\mathrm{B}^{\tau_{0}, n}$ consists of a disjoint union of quadrilaterals with circular sides. After renormalization, the part of the boundary of $\mathrm{B}^{\tau_{0}, \mathrm{n}}$ corresponding to $\left\{|\operatorname{Im}(z)|=\tau_{0}\right\}$ is the horizontal part of the boundary, while the part of the boundary corresponding to $\{|\operatorname{Re}(z)|=\mathfrak{n}\}$ is the vertical part of the boundary. We make a fixed choice of the conjugating maps, $\mathrm{H}_{i}, i=1, \ldots, \mathrm{q}$, and we fix a choice of the parameter $\tau>\tau_{0}$ in the above construction. We may deform all the loops $\widehat{\alpha}_{i}$ and $\widehat{\alpha}_{i}^{\prime}$, within $\Omega^{+}(\widehat{G})$ to an equivalent fundamental set of loops, with the same geometric generators $\widehat{A}_{1}, \ldots, \widehat{A}_{g}$, so that, after appropriate renormalization, each connected component of each of the deformed loops appears, in each component of the complement of the flat part of $\mathrm{B}^{\tau}$, as a pair of half-infinite Euclidean vertical lines, one in $\{\operatorname{Im}(z) \geq \tau\}$, the other in $\{\operatorname{Im}(z) \leq-\tau\}$, both with the same real part (the technical details of such a deformation can be found in [7). We call such a deformed loops the vertical projection loops. These vertical projection loops, which we still denoting as $\widehat{\alpha}_{1}, \ldots, \widehat{\alpha}_{\mathrm{g}}^{\prime}$, yields a fundamental set of loops, $\alpha_{1}^{\tau}, \ldots, \alpha_{g}^{\prime \tau}$ for the generators $A_{1}^{\tau}, \ldots, A_{g}^{\tau}$ of the Schottky group $G^{\tau}$.

\section{(III) The relative conical neighborhood}

The relative conical neighborhood of $\widehat{G}$ is to be defined as the set of all marked Schottky groups $\mathrm{G}^{\tau}=\left\langle\mathrm{A}_{1}^{\tau}, \ldots, \mathrm{A}_{\mathrm{g}}^{\tau}\right\rangle$, with the fundamental set of loops $\alpha_{1}^{\tau}, \ldots, \alpha_{g}^{\prime \tau}$, as constructed above.

Remark 2. Recall that we are assuming that $\infty$ is an interior point of the flat part corresponding to $\tau_{0}$, and $f^{\tau}(z)=z+O\left(|z|^{-1}\right)$ near $\infty$. As, with these normalizations, $f^{\tau} \rightarrow$ I uniformly on compact subsets of $\Omega(\widehat{G})$, we obtain that $\mathrm{G}^{\tau} \rightarrow \widehat{\mathrm{G}}$ algebraically. It now follows, from the Jørgensen-Marden criterion [10, that $\mathrm{G}^{\mathcal{\tau}} \rightarrow \widehat{\mathrm{G}}$ geometrically and that each relative conical neighborhood contains infinitely many distinct marked Schottky groups. It is also easy to see, as in [19, that, for each primitive parabolic element $\widehat{P} \in \widehat{G}$, as $\tau \rightarrow \infty$, there is a corresponding geodesic on $S^{\tau}=\Omega\left(G^{\tau}\right) / G^{\tau}$ whose length tends to zero. It follows that each relative conical neighborhood of a noded Schottky group contains Schottky groups representing infinitely many distinct Riemann surfaces.

\subsubsection{Pinchable loops of Schottky groups}

Let $G$ be a Schottky group of rank $g \geq 2$, with generators $A_{1}, \ldots, A_{g}$, and let $\pi: \Omega(G) \rightarrow S$ be a regular covering with deck group $\mathrm{G}$.

\section{(IV) Pinchable loops}

Let $\gamma_{1}, \ldots, \gamma_{q}$ be a set of simple disjoint geodesics loops on $\mathrm{S}$. Each $\gamma_{j}$ corresponds to a conjugacy class of a cyclic subgroup of $\mathrm{G}$ (including the trivial subgroup) by the lifting under $\pi$; let $\left\langle W_{\mathbf{j}}\right\rangle$ be a representative of such a class. If these $\mathrm{q}$ cyclic subgroups are non-trivial, they are pairwise non-conjugated in $G$ and the generators $W_{j}$ are non-trivial powers in $G$ (i.e., there is no $T_{j} \in G$ so 
that $W_{j}=T_{j}^{m_{j}}$ for some $\left.m_{j} \geq 2\right)$, then we say that this set of geodesics is pinchable in $G$.

Remark 3. (1) It was shown in 20] (see also Yamamoto 25]) that if $\gamma_{1}, \ldots, \gamma_{\mathrm{q}}$ is a set of pinchable simple disjoint geodesics loops on $S$ in $G$, defined by the words $W_{1}, \ldots, W_{q}$, as above, then there is a noded Schottky group $\widehat{G}$, and there is an isomorphism $\psi: G \rightarrow \widehat{G}$, where $\psi\left(W_{1}\right), \ldots, \psi\left(W_{q}\right)$, and their powers and conjugates, are exactly the parabolic elements of $\widehat{\mathrm{G}}$. More precisely, it was shown in 20] that there is a path in Schottky space, $\mathcal{S}_{\mathrm{alg}}$, which converges to a set of generators for $\widehat{\mathrm{G}}$, along which the lengths of the geodesics, $\gamma_{1}, \ldots, \gamma_{q}$, all tend to zero. (2) On the other direction, let us consider a noded Schottky group $\widehat{\mathrm{G}}$ of rank $\mathrm{g} \geq 2$, with a set of geometrical generators $\widehat{A}_{1}, \ldots, \widehat{A}_{g}$ and corresponding fundamental set of loops $\widehat{\alpha}_{1}, \ldots, \widehat{\alpha}_{g}^{\prime}$. Let $G^{\tau}$ be a Schottky group of rank $g$ with fundamental set of loops $\alpha_{1}^{\tau}, \ldots, \alpha_{g}^{\prime \tau}$ and generators $A_{1}^{\tau}, \ldots, A_{g}^{\tau}$, in a relative conical neighborhoodof $\widehat{G}$ as previously described in Section 2.4.1. Let $S=\Omega\left(G^{\tau}\right) / G^{\tau}$ be the closed Riemann surface of genus $g$ represented by $G^{\tau}$, and let $V_{i} \subset S$ be the projection of $\alpha_{i}^{\tau}$, $i=1, \ldots, g$. Then $V_{1}, \ldots, V_{g}$ is a set of $g$ homologically independent simple disjoint loops on $S$. Let $\psi: G^{\tau} \rightarrow \widehat{G}$ be the isomorphism defined by $A_{i}^{\tau} \mapsto \widehat{A}_{i}, i=1, \ldots, g$. The elements of $G^{\tau}$ which are sent to parabolic elements of $\widehat{\mathrm{G}}$ are called the pinched elements of $\mathrm{G}^{\tau}$. There are simple disjoint geodesics $\gamma_{1}, \ldots, \gamma_{q}$ on $S$, defined by pinched elements of $G^{\tau}$, given by the words $W_{1}, \ldots, W_{q}$ in the generators $A_{1}^{\tau}, \ldots, A_{g}^{\tau}$, so that every parabolic element of $\widehat{G}$ is a power of a conjugate of one of their $\psi$-image. It happens that this collection of loops $\gamma_{1}, \ldots, \gamma_{\mathrm{q}}$ is a set of pinchable geodesics of $\mathrm{G}^{\tau}$. The construction in [19] shows that we can choose the above parameter $\tau$ so that the $\gamma_{i}$ are all arbitrarily short.

Proposition 1 (7]). Every non-empty set of $\mathrm{k}<3 \mathrm{~g}-3$ pinchable geodesics is contained in a maximal set of $3 \mathrm{~g}-3$ pinchable geodesics.

\section{(V) Valid sets of fundamental loops and their complexity}

Let $\gamma_{1}, \ldots, \gamma_{\mathrm{q}} \subset \mathrm{S}$ be a pinchable set of geodesics in G. Set $\widehat{S}^{+}$the stable Riemann surface obtained from $S$ by pinching these $q$ geodesics; it consists of a finite number of compact Riemann surfaces, called parts, which are joined together at a finite number of nodes. Also, let $\widehat{G}$ be the noded Schottky group obtained from $\mathrm{G}$ by pinching these $\mathrm{q}$ geodesics.

Let $V_{1}, \ldots, V_{g}$, be a fundamental set of loops for $G$ on $S$ (that is, the components of the lifting of these loops under $\pi$ are simple loops and such a lifting set of loops contains a fundamental set of loops for G) and let $\widehat{V}_{1}, \ldots, \widehat{V}_{g}$ be the corresponding loops on $\widehat{S}^{+}$obtained by pinching $\gamma_{1}, \ldots, \gamma_{q}$. We observe that the lifts of the $\widehat{V}_{i}$ to $\Omega^{+}(\widehat{\mathrm{G}})$ are all loops, but they are generally not disjoint and they need not to be simple. There are certainly some number of these lifts passing through each parabolic fixed point, and some of them might pass more than once through the same parabolic fixed point. The set of loops, $V_{1}, \ldots, V_{g}$, is called a valid set of fundamental loops for $\gamma_{1}, \ldots, \gamma_{q}$, if every lift of every $\widehat{V}_{i}$ to $\Omega^{+}(\widehat{G})$ is a simple loop; that is, it passes at most once through each parabolic fixed point (i.e., the set of loops, $\widehat{V}_{1}, \ldots, \widehat{V}_{g}$, forms a fundamental set of loops for $\widehat{G}$ on 
$\widehat{\mathrm{S}}^{+}$). We note that there are exactly q equivalence classes of parabolic fixed points in $\widehat{\mathrm{G}}$, one for each of the loops $\gamma_{i}$.

Proposition $2(4,7])$. There is at least one valid set of fundamental loops $\mathrm{V}_{1}, \ldots, \mathrm{V}_{\mathrm{g}}$, for every set of pinchable geodesics, $\gamma_{1}, \ldots, \gamma_{\mathrm{q}}$.

\section{(VI) The complexity}

Let us now consider a valid set of fundamental loops, $V_{1}, \ldots, V_{g}$, for a set of pinchable geodesics $\gamma_{1}, \ldots, \gamma_{\mathbf{q}}$. We can deform the $V_{i}$ on $S$ so that they are all geodesics. Then the geometric intersection number, $V_{i} \bullet \gamma_{j}$, of $V_{i}$ with $\gamma_{j}$ is well defined; it is the number of points of intersection of these two geodesics. Looking on the corresponding noded surface $\widehat{S}^{+}, V_{i} \bullet \gamma_{j}$ is the number of times the curve $\widehat{V}_{i}$ obtained from $V_{i}$ by contracting $\gamma_{j}$ to a point, passes through that point (node). The complexity of $V_{1}, \ldots, V_{g}$, with respect to $\gamma_{1}, \ldots, \gamma_{q}$, is given by

$$
\Xi\left(\gamma_{1}, \ldots, \gamma_{q} ; V_{1}, \ldots, V_{g}\right)=\max _{1 \leq j \leq q} \sum_{i=1}^{g} v_{i} \bullet \gamma_{j}
$$

and the complexity $\Xi\left(\gamma_{1}, \ldots, \gamma_{\mathrm{q}}\right)$ is the minimum of $\Xi\left(\gamma_{1}, \ldots, \gamma_{\mathrm{q}} ; \mathrm{V}_{1}, \ldots, \mathrm{V}_{\mathrm{g}}\right)$, where the minimum is taken over all valid sets of fundamental loops. If $\Xi\left(\gamma_{1}, \ldots, \gamma_{q}\right) \geq n$, then, for every valid fundamental set $\mathrm{V}_{1}, \ldots, \mathrm{V}_{\mathrm{g}}$, there is a node $\mathrm{P}$ on $\mathrm{S}^{+}$so that the total number of crossings of $\mathrm{P}$ by $\widehat{V}_{1}, \ldots, \widehat{V}_{g}$ is at least $n$.

Proposition 3 ([7]). Let $\mathrm{g} \geq 2$ and $\mathrm{G}$ be a Schottky group of rank $\mathrm{g}$. For each positive integers $\mathrm{n}$ there are only finitely many topologically distinct maximal (i.e. $\mathrm{q}=3 \mathrm{~g}-3$ ) pinchable set of geodesics in $\mathrm{G}$ and complexity $\mathrm{n}$. In particular, there are infinitely many topologically distinct maximal noded Schottky groups of rank $\mathrm{g}$ and there are only finitely many topologically distinct maximal neoclassical noded Schottky groups in each rank $\mathrm{g}$.

\section{(VII) Sufficiently complicated pinchable sets of geodesics}

Now, we consider a maximal set of pinchable geodesics in $\mathrm{G}$, say $\gamma_{1}, \ldots, \gamma_{3 g-3}$; so $\widehat{\mathrm{G}}$ is a maximal noded Schottky group. Observe that $\widehat{G}$ is rigid, and that every part of $\mathrm{S}^{+}$is a sphere with three distinct nodes. Also, every connected component $\Delta \subset \Omega(\widehat{\mathrm{G}})$ is a Euclidean disc $\Delta$, where $\Delta / \operatorname{Stab}(\Delta)$ is a sphere with three punctures; the three punctures correspond to the three nodes of the corresponding part of $\widehat{S}^{+}$.

Let $V_{1}, \ldots, V_{g}$ be a valid set of fundamental loops on $S$ for the given set of pinchable geodesics (the existence is given by Proposition 2), and let $\widehat{V}_{1}, \ldots, \widehat{V}_{g}$ be the corresponding loops on $\widehat{S}^{+}$. For each $i=1, \ldots, g$, the intersection of a lifting of $\widehat{V}_{i}$ with a component of $\widehat{G}$ (i.e., a connected component of $\Omega(\widehat{G}))$ is called a strand of that lifting $\widehat{V}_{i}$. Similarly, the loops $\widehat{V}_{1}, \ldots, \widehat{V}_{g}$ appear on the corresponding parts of $\widehat{\mathrm{S}}^{+}$as collections of strands connecting the nodes on the boundary 
of each part. There are two possibilities for these strands; either a strand connects two distinct nodes on some part, or it starts and ends at the same node. Since the loops $V_{1}, \ldots, V_{g}$ are simple and disjoint, there are at most three sets of parallel strands of the $\widehat{V}_{i}$ in each part; that is, there are at most three sets of strands, where any two strands in the same set are homotopic arcs with fixed endpoints at the nodes. We regard each of these sets of strands on a single part as being a superstrand, so that there are at most 3 superstrands on any one part. We next look in some component $\Delta$ of $\Omega(\widehat{\mathrm{G}})$, and look at a parabolic fixed point $x$ on its boundary, where $x$ corresponds to the node $\mathrm{N}$ on the part $S_{i}$ of $\widehat{S}^{+}$. In general, there will be infinitely many liftings of superstrands emanating from $\mathrm{x}$ in $\Delta$, but, modulo $\operatorname{Stab}(\Delta)$ there are only finitely many. In fact, there are at most 4 such liftings of superstrands emanating from $x$. If there is exactly one superstrand on $S_{i}$ with one endpoint at $N$, and the other endpoint at a different node, then modulo $\operatorname{Stab}(\Delta)$ there will be exactly the one lifting of this superstrand emanating from $x$. If there is only one superstrand on $S_{i}$ with both endpoints at the same node $\mathrm{N}$, then this superstrand has two liftings starting at $x$, one in each direction; so, in this case, we see two lifts of superstrands modulo $\operatorname{Stab}(\Delta)$ emanating from $x$. It follows that, modulo $\operatorname{Stab}(\Delta)$, we can have $0,1,2,3$ or 4 liftings of superstrands starting at $x$. We note that these liftings of superstrands all end at distinct parabolic fixed points on the boundary of $\Delta$. We say that the fundamental set of loops, $\widehat{V}_{1}, \ldots, \widehat{V}_{p}$ is sufficiently complicated if there are two (different) lifts $\widehat{\alpha}_{i}$ and $\widehat{\alpha}_{j}$, of some $\widehat{V}_{i}$ and some not necessarily distinct $\widehat{V}_{j}$, respectively, so that $\widehat{\alpha}_{i}$ and $\widehat{\alpha}_{j}$ both pass through the parabolic fixed point $z_{1}$, into a component $\Delta_{1}$ of $\widehat{G}$, then both travel through $\Delta_{1}$ to the same parabolic fixed point on its boundary, $z_{2}$, and into another component $\Delta_{2}$, which they again traverse together to the same boundary point, $z_{3}$, necessarily a parabolic fixed point, where they enter $\Delta_{3}$, and they leave $\Delta_{3}$ at different parabolic fixed points.

\subsubsection{Sufficiently complicated noded Schottky groups}

A maximal noded Schottky group $\widehat{G}$ is sufficiently complicated if every set of valid fundamental loops on $\widehat{S}^{+}$is sufficiently complicated. We note that (keeping the notation of last section), inside $\Delta_{1}, \widehat{\alpha}_{i}$ and $\widehat{\alpha}_{j}$ are disjoint; they both enter $\Delta_{1}$ at the same point, and they both leave $\Delta_{1}$ at the same point; hence they cannot both be circles. In [7] the following result, stating a sufficient condition in terms of the complexity for a maximal noded Schottky group to be sufficiently complicated, was obtained.

Theorem 1 ([7]). If a maximal noded Schottky group $\widehat{\mathrm{G}}$ has complexity at least 11 , then it is sufficiently complicated.

The previous theorem, together Proposition 3 asserts the existence of infinitely many topologically different sufficiently complicated maximal noded Schottky groups in every rank $\mathrm{g} \geq 2$. The following result states sufficient conditions for a Schottky group to be non-classical.

Theorem 2 ([7]). Let $\widehat{\mathrm{G}}$ be a maximal noded Schottky group. 
(1) If $\widehat{\mathrm{G}}$ is sufficiently complicated, then, for $\tau$ sufficiently large, the Schottky group $\mathrm{G}^{\tau}$ in the relative conical neighborhood of $\widehat{\mathrm{G}}$ is non-classical.

(2) If $\mathrm{S}^{+}=\Omega^{+}(\widehat{\mathrm{G}}) / \widehat{\mathrm{G}}$ is the stable Riemann surface as shown in figure Q, then $\widehat{\mathrm{G}}$ is sufficiently complicated.

\section{Explicit construction of a sufficiently complicated noded Schottky group}

In this section we construct explicitly a noded Schottky group as in part (2) of Theorem 2, so part (1) of the same theorem asserts that any Schottky group sufficiently near to $\widehat{G}$ is necessarily non-classical.

\subsection{A family Schottky groups of rank three}

Let $L_{0}$ be the unit circle, $L_{1}$ be the real line, $L_{2}$ be the line through the points 0 and $w_{0}=e^{\pi i / 3}$, and set (see Figure 7)

$$
\mathcal{F}=\left\{(p, r): 1 / 2<p<1,0<r<r^{*}(p):=\frac{\sqrt{1+p^{2}+p^{4}}+p^{2}-1}{\sqrt{3} p}\right\} .
$$

For each $(p, r) \in \mathcal{F}$ we set $L_{3}$ to be the circle with center at 0 and radius $r$ and $L_{4}$ to be the circle orthogonal to $L_{0}$, intersecting $L_{1}$ at the points $p$ and $1 / p$ with angle $\pi / 3$ (see Figure 3 ). The circle $L_{4}$ has its center at $c=\left(\frac{1+p^{2}}{2 p}\right)+\frac{i}{\sqrt{3}}\left(\frac{1-p^{2}}{2 p}\right)$ and it has radius $R=\frac{2}{\sqrt{3}}\left(\frac{1-p^{2}}{2 p}\right)$. The condition $p>1 / 2$ asserts that $L_{2}$ and $L_{4}$ are disjoint (tangency occurs when $p=1 / 2$ ) and the condition $r<r^{*}(p)$ asserts that $L_{3}$ and $L_{4}$ are disjoint (tangency occurs when $r=r^{*}(p)$ ). Let $\tau_{j}$ be the reflection on $L_{j}$, for $j=0,1,2,3,4$, so

$$
\tau_{0}(z)=1 / \bar{z}, \quad \tau_{1}(z)=\bar{z}, \quad \tau_{2}(z)=w^{2} \bar{z}, \quad \tau_{3}(z)=r^{2} / \bar{z}, \quad \tau_{4}(z)=\frac{c \bar{z}-1}{\bar{z}-\bar{c}}
$$

and let $\mathrm{K}_{\mathrm{r}, \mathrm{p}}=\left\langle\tau_{0}, \tau_{1}, \tau_{2}, \tau_{3}, \tau_{4}\right\rangle$. It turns out that $\mathrm{K}_{\mathrm{r}, \mathrm{p}}$ is an extended Kleinian group with connected region of discontinuity $\Omega_{r, p}$ and so that $\Omega_{r, p} / K_{r, p}$ is a closed disc with 5 branch values, of orders 2, 2, 2, 2 and 3, on its border. As a consequence of the Klein-Maskit combination theorems [18], the group $\mathrm{K}_{\mathrm{r}, \mathrm{p}}$ has no parabolic transformations, its limit set is a Cantor set and it is geometrically finite. If we set $W=\tau_{2} \tau_{1}, J=\tau_{0} \tau_{1}$ and $L=\tau_{1} \tau_{4}$, then $W^{3}=L^{3}=J^{2}=(W J)^{2}=$ $(\mathrm{LJ})^{2}=1$.

$$
\begin{aligned}
\text { Now, if } A_{1}=L^{-1} W^{-1} & =\tau_{4} \tau_{2}, A_{2}=\tau_{1} A_{1} \tau_{1}, \text { and } A_{3}=\tau_{0} \tau_{3}, \text { so } \\
A_{1}(z) & =\frac{c w_{0} z-1}{w_{0} z-\bar{c}}, \quad A_{2}(z)=\frac{\bar{c} w_{0}^{2} z-1}{w_{0}^{2} z-c}, \quad A_{3}(z)=r^{2} z,
\end{aligned}
$$




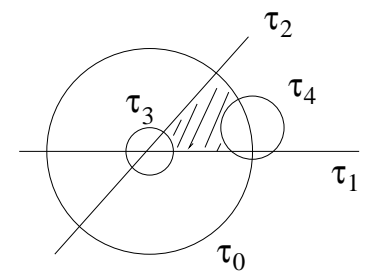

Figure 3 - A set of lines and circles

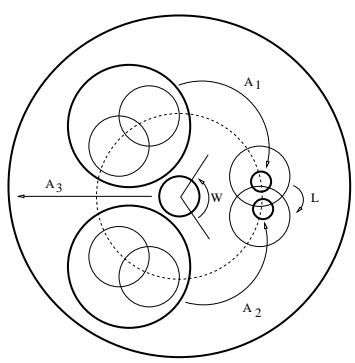

Figure 4-The Schottky group $\mathrm{G}_{\mathrm{r}, \mathrm{p}}$ of rank 3: the six darkest loops are a fundamental set of loops

then we set $G_{r, p}=\left\langle A_{1}, A_{2}, A_{3}\right\rangle$. In Figure 4 we show the situation for values of $p$ near to 1 and $r$ near to 0 ; in which case $G_{r, p}$ turns out to be a classical Schottky group of rank three.

Lemma 1. If $(\mathrm{p}, \mathrm{r}) \in \mathcal{F}$, then $\mathrm{G}_{\mathrm{r}, \mathrm{p}}$ is a Schottky group of rank three.

Proof. It can be seen that $\mathrm{G}_{\mathrm{r}, \mathrm{p}}$ is a finite index normal subgroup of $\mathrm{K}_{\mathrm{r}, \mathrm{p}}$ and

$$
\mathrm{K}_{\mathrm{r}, \mathrm{p}} / \mathrm{G}_{\mathrm{r}, \mathrm{p}}=\left\langle\tau_{0}: \tau_{0}^{2}=1\right\rangle \times\left\langle\tau_{1}, \tau_{2}: \tau_{1}^{2}=\tau_{2}^{2}=\left(\tau_{2} \tau_{1}\right)^{3}=1\right\rangle \cong \mathbb{Z}_{2} \oplus \mathrm{D}_{3} .
$$

In particular, $G_{r, p}$ has the same region of discontinuity as for $K_{r, p}$ (so a function group), it is geometrically finite and does not have parabolic transformations. As any of the elliptic transformations of $K_{r, p}$ goes into an element of the same order in the quotient $K_{r, p} / G_{r, p}$, we also have that $G_{r, p}$ is torsion free. Now, as a consequence of the classification of function groups 15, 16, the group $\mathrm{G}_{\mathrm{r}, \mathrm{p}}$ is a Schottky group of rank three (in Figure 4 there is shown a fundamental set of loops).

The closed Riemann surface $S_{r, p}=\Omega_{r, p} / G_{r, p}$ of genus 3 admits the group $\mathbb{Z}_{2} \oplus D_{3}$ as a group of conformal/anticonformal automorphisms. On $S_{r, p}$ we have simple closed curves $\gamma_{1}, \ldots, \gamma_{6}$ which are pinchable (see Figures 5 and 6 ) with respect to the Schottky group $\mathrm{G}_{\mathrm{r}, \mathrm{p}}$; these pinchable curves correspond to the conjugacy classes of cyclic groups of $G_{r, p}$ as follows:

$\gamma_{1}$ corresponds to $A_{2}^{-1} ; \gamma_{2}$ corresponds to $A_{1} ; \gamma_{3}$ corresponds to $A_{1}^{-1} A_{2}$; $\gamma_{4}$ corresponds to $A_{1}^{-1} A_{2} A_{3}^{-1} A_{2}^{-1} A_{1} A_{3} ; \gamma_{5}$ corresponds to $A_{2}^{-1} A_{3}^{-1} A_{2} A_{3}$; $\gamma_{6}$ corresponds to $A_{1} A_{3}^{-1} A_{1}^{-1} A_{3}$. 


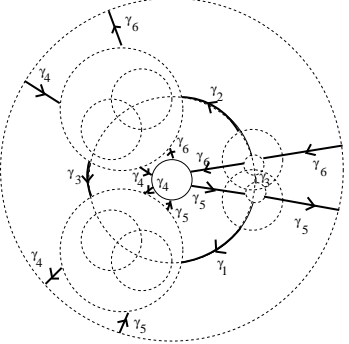

Figure 5-A set of pinchable curves seen at the Schottky uniformization

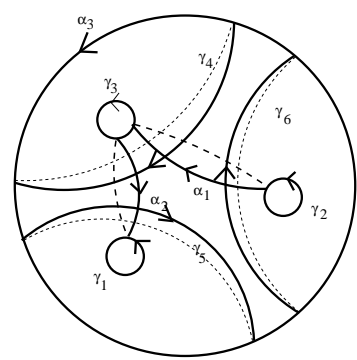

Figure 6-A set of pinchable curves seen on the Riemann surface $S_{r, p}$

\subsection{A sufficiently complicated noded Schottky groups of rank three}

To obtain the desired noded Schottky group, we need to move the pair $(p, r) \in \mathcal{F}$ to some point in the boundary in order to have that the loxodromic transformations $A_{2}^{-1}, A_{1}, A_{1}^{-1} A_{2}$, $A_{1}^{-1} A_{2} A_{3}^{-1} A_{2}^{-1} A_{1} A_{3}, A_{2}^{-1} A_{3}^{-1} A_{2} A_{3}$ and $A_{1} A_{3}^{-1} A_{1}^{-1} A_{3}$ are transformed into parabolic transformations. As the order three automorphism $W$ permutes cyclically $\gamma_{1}, \gamma_{2}, \gamma_{3}$ and also $\gamma_{4}, \gamma_{5}, \gamma_{6}$, we only need to take care of $A_{1}$ and $A_{1} A_{3}^{-1} A_{1}^{-1} A_{3}$. First, in order to transform $A_{1}$ into a parabolic transformation we only need to have $\tau_{4}\left(w_{0}\right)=w_{0}$, equivalently, that the circle $L_{4}$ is tangent to the line $L_{2}$ at $w_{0}$. This happens exactly when $p=1 / 2$. Now, assuming $p=1 / 2$, in order for $A_{1} A_{3}^{-1} A_{1}^{-1} A_{3}$ to be a parabolic transformation we only need to have tangency of the circle $L_{3}$ with $\mathrm{L}_{4}$, that is, $r=r^{*}(1 / 2)=\frac{\sqrt{7}-\sqrt{3}}{2}$. The group $\mathrm{G}_{\mathrm{r}^{*}(1 / 2), 1 / 2}$ turns out to be a noded Schottky group that uniformizes a stable Riemann surface as shown in Figure 2 and, by (2) in Theorem 2 , it is a sufficiently complicated noded Schottky group.

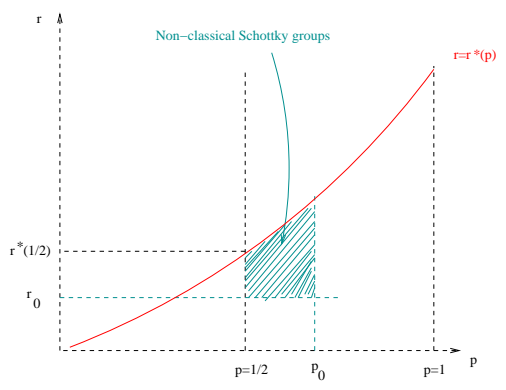

Figure 7 - The region $\mathcal{F}$ and the filled part for the non-classical Schottky groups

\subsection{Non-classical Schottky groups of rank three}

By (1) in Theorem 2, there exist $\left(p_{0}, r_{0}\right) \in \mathcal{F}$ with the property that if $(p, r) \in \mathcal{F}, 1 / 2<p<p_{0}$ and $r_{0}<r<r^{*}(1 / 2)$, then $G_{r, p}$ is a non-classical Schottky group of rank three (see filled part region in Figure 7). Moreover, each of these Schottky groups is contained in a Kleinian group $\mathrm{K}_{r, p}$ 
as a finite index normal subgroup with $\mathrm{K}_{\mathrm{r}, \mathrm{p}} / \mathrm{G}_{\mathrm{r}, \mathrm{p}} \cong \mathbb{Z}_{2} \oplus \mathrm{D}_{3}$, in other words, the closed Riemann surfaces $S_{r, p}=\Omega\left(G_{r, p}\right) / G_{r, p}$ admit a group of conformal automorphisms isomorphic to $\mathbb{Z}_{2} \oplus D_{3}$. The family of these Riemann surfaces degenerates to a stable Riemann surface $S_{r^{*}(1 / 2), 1 / 2}$ as Figure 2 keeping the above group of automorphisms invariant. 


\section{References}

[1] A. I. Bobenko. Schottky uniformization and finite-gap integration, Soviet Math. Dokl. 36 No. 1 (1988), 38-42 (transl. from Russian: Dokl. Akad. Nauk SSSR, 295, No.2 (1987)).

[2] J. Button. All Fuchsian Schottky groups are classical Schottky groups. Geometry \& Topology Monographs. Volume 1: The Epstein birthday schrift (1998), 117-125.

[3] V. Chuckrow. Schottky groups and limits of Kleinian groups. Bull. Amer. Math. Soc. 73 No. 1 (1967), 139-141.

[4] R. A. Hidalgo. The noded Schottky space. London Math. Soc. 73 (1996), 385-403.

[5] R. A . Hidalgo. Noded Fuchsian groups. Complex Variables 36 (1998), 45-66.

[6] R. A. Hidalgo. Towards a proof of the classical Schottky uniformization conjecture. https://arxiv.org/pdf/1709.09515.pdf

[7] R. A. Hidalgo and B. Maskit. On neoclassical Schottky groups. Trans. of the Amer. Math. Soc. 358 (2006), 4765-4792.

[8] Y. Hou. On smooth moduli space of Riemann surfaces. (2016). https://arxiv.org/pdf/1610.03132.pdf

[9] Y. Hou. The classification of Kleinian groups of Hausdorff dimensions at most one. (2016). https://arxiv.org/pdf/1610.03046.pdf

[10] T. Jørgensen and A. Marden. Algebraic and geometric convergence of Kleinian groups. Math. Scand. 66 (1990), 47-72.

[11] P. Koebe. Über die Uniformisierung der Algebraischen Kurven II. Math. Ann. 69 (1910), 1-81.

[12] I. Kra and B. Maskit. Pinched two component Kleinian groups. In Analysis and Topology, pages 425-465. World Scientific Press, 1998.

[13] C. McMullen. Complex Dynamics and Renormalization. Annals of Mathematical Studies 135, Princeton University Press, (1984).

[14] A. Marden. Schottky groups and circles. In Contributions to Analysis (a collection of papers dedicated to Lipman Bers), 273-278, Academic Press, 1974.

[15] B. Maskit. On the classification of Kleinian Groups I. Koebe groups. Acta Math. 135 (1975), 249-270.

[16] B. Maskit. On the classification of Kleinian Groups II. Signatures. Acta Math. 138 (1976), $17-42$. 
[17] B. Maskit. Remarks on m-symmetric Riemann surfaces. Contemporary Math. 211 (1997), 433-445.

[18] B. Maskit. On Klein's combination theorem IV. Trans. Amer. Math. Soc. 336 (1993),265-294.

[19] B. Maskit. On free Kleinian groups. Duke Math. J. 48 (1981),755-765.

[20] B. Maskit. Parabolic elements in Kleinian groups. Annals of Math. 117 (1983), 659-668.

[21] N. Purzitsky. Two-Generator Discrete Free Products. Math. Z. 126 (1972), 209-223.

[22] H. Sato. On a paper of Zarrow. Duke Math. J. 57 (1988), 205-209.

[23] M. Seppälä. Myrberg's numerical uniformization of hyperelliptic curves. Ann. Acad. Scie. Fenn. Math. 29 (2004), 3-20.

[24] J. P. Williams. Classical and non-classical Schottky groups. Doctoral Thesis, University of Southampton, School of Mathematics (2009), 125pp.

[25] Hiro-o Yamamoto. Sqeezing deformations in Schottky spaces. J. Math. Soc. Japan 31 (1979), $227-243$.

[26] Hiro-o Yamamoto. An example of a non-classical Schottky group. Duke Math. J. 63 (1991), 193-197.

[27] R. Zarrow. Classical and non-classical Schottky groups. Duke Math. J. 42 (1975), 717-724. 